\title{
Clasificación trófica de insectos acuáticos en ocho quebradas protegidas de la ecorregión cafetera colombiana
}

\author{
Ana M. Chará-Serna ${ }^{1 *}$, Julián D. Chará ${ }^{1,2}$, María del Carmen Zúñiga ${ }^{1,3}$, Gloria X. Pedraza $^{1}$, Lina P. Giraldo $^{1}$ \\ ${ }^{1}$ Centro para la Investigación en Sistemas Sostenibles de Producción Agropecuaria - CIPAV. Carrera 25 No. 6-62 Cali, Colombia. \\ ${ }^{2}$ Centro de Investigaciones y Estudios en Biodiversidad y Recursos Genéticos, CIEBREG, P.O.B. 97, Pereira, Colombia. \\ ${ }^{3}$ Universidad del Valle. Departamento de Biología, Grupo de Investigaciones Entomológicas. Calle 13 No. 100-00 Cali, Colombia. \\ *ana@cipav.org.co
}

Recibido: 01-12-2009; Aceptado: 25-04-2010

\begin{abstract}
Resumen
Objetivo. Determinar la estructura trófica del ensamblaje de insectos acuáticos asociados a ocho quebradas de la ecorregión cafetera colombiana. Materiales y métodos. Se colectaron insectos acuáticos en ocho quebradas protegidas por corredores de bosque en la cuenca del río La Vieja. Los taxa encontrados fueron asignados a grupos dietarios con base en una clasificación regional, generada a partir de la revisión del contenido estomacal de los insectos, en quebradas boscosas de la cuenca del río Otún. Resultados. En total se colectaron 2019 individuos distribuidos en 73 grupos taxonómicos, de los cuales 60 fueron clasificados en grupos dietarios. Los colectores fueron el grupo más abundante (55\%), seguido por los fragmentadores (31\%) y los depredadores (10\%). Los raspadores sólo representaron el $0,05 \%$ de la muestra y el 3,95\% restante no pudo ser clasificado por falta de información. Conclusiones. La dominancia de colectores y fragmentadores refleja la importancia de la materia orgánica particulada gruesa (hojarasca), como recurso alimentario para la entomofauna. La similitud de la estructura trófica entre esta comunidad y otras estudiadas en quebradas similares, sugiere la posibilidad de un patrón común para las quebradas andinas. Se evidenció la falta de información sobre ecología trófica de insectos acuáticos tropicales; 50\% de los taxa colectados no presentaron reportes de este tipo en el trópico y para el $20 \%$ no existe información en el trópico ni en la zona templada.
\end{abstract}

Palabras clave: insectos acuáticos, grupos dietarios, estructura trófica, quebradas andinas, ecosistemas tropicales.

\begin{abstract}
Trophic classification of aquatic insects in eight sheltered streams of the Colombian coffee ecoregion. Objective. To determine the trophic structure of the aquatic insect assembly associated to eight streams in the Colombian coffee-growing ecoregion. Materials and methods. Aquatic insects were collected in eight forested streams located in La Vieja river basin. The taxa collected were assigned to dietary groups according to a regional classification based on the gut content analysis of aquatic insects associated to forested streams of the Otún river basin. Results. 2019 individuals belonging to 73 taxa were collected and 60 were classified into dietary groups. The most abundant group was collectors (55\%), followed by shredders (31\%) and predators (10\%). Scrapers represented only $0.05 \%$ of the sample and the remaining 3,95\% could not be classified due to lack of information. Conclusions. The dominance of collectors and shredders reveals the importance of coarse particulate organic matter (leaf litter) as a food resource for the insect fauna. Similarities between the trophic structure of this community and other communities studied in similar streams, suggest the possibility of a common pattern for Andean streams. This study evidenced the lack of knowledge on trophic ecology of tropical aquatic insects; $50 \%$ of the taxa collected did not have this kind of information for the tropics and $20 \%$ had no information neither for the tropics nor temperate zones.
\end{abstract}

Key words: andean streams, aquatic insects, dietary groups, trophic structure, tropical ecosystems. 


\section{Resumo}

Classificação trófica de insetos aquáticos em oito córregos protegidos da ecorregião cafetera colombiana. Objetivo. Determinar a estrutura trófica da assembléia de insetos aquáticos associados a oito córregos da ecorregião cafetera colombiana. Materiais e métodos. Foram coletados insetos aquáticos em oito córregos protegidos por corredores florestais na bacia do rio La Vieja. Os taxa encontrados foram designados a grupos de dieta baseados numa classificação regional, gerada a partir da análise do conteúdo estomacal dos insetos, em córregos da bacia do rio Otún. Resultados. Foram coletados 2.019 indivíduos pertencentes a 73 grupos taxonômicos, dos quais 60 foram classificados em grupos alimentares. Os coletores foram o grupo mais abundante (55\%), seguido por fragmentadores (31\%) e predadores (10\%). Os raspadores representaram apenas $0,05 \%$ da amostra e os restantes 3,95\% não puderam ser classificados devido à falta de informação. Conclusões. O predomínio de coletores e fragmentadores reflete a importância da matéria orgânica particulada grossa (serrapilheira), como recurso alimentar para a fauna de insetos. A semelhança entre a estrutura trófica desta comunidade e outras estudadas em córregos similares, sugerem a possibilidade de um padrão comum para córregos andinos. Este estudo revelou a falta de informação sobre a ecologia alimentar de insetos aquáticos tropicais, 50\% dos taxa coletados não apresentaram nenhum registro deste tipo no trópico, e para o $20 \%$ não há informação nem nos trópicos nem na zona temperada.

Palavras-chave: insetos aquáticos, grupos tróficos, estrutura trófica, córregos andinos, ecossistemas tropicais.

\section{Introducción}

Las relaciones tróficas son un elemento importante en la estructura de las comunidades de insectos acuáticos porque son determinantes en todos los aspectos de la vida de los invertebrados (ciclos de vida, elección de hábitat, comportamiento, predación) y en procesos ecológicos como la circulación de nutrientes (1). Por esta razón la ecología trófica de la entomofauna acuática ha sido ampliamente trabajada en quebradas de la zona templada (e.g. 2-4). Sin embargo, existen pocos estudios ecológicos sobre insectos acuáticos en ecosistemas tropicales y por lo general, estos estudios determinan los gremios tróficos de los taxa basados en clasificaciones desarrolladas para zonas templadas (e.g. 5-7), como la de Merritt y Cummins (2). Se ha comprobado en diferentes investigaciones que esta aproximación puede ser inexacta, puesto que los taxa clasificados en un grupo trófico determinado en ecosistemas templados, no necesariamente presentan los mismos hábitos dietarios en el trópico (8-10).

En Colombia las quebradas andinas pequeñas son ecosistemas relevantes, como fuentes de agua para actividades rurales y urbanas. Aunque son pocas las investigaciones publicadas sobre estos ambientes, ya se ha realizado un primer esfuerzo para clasificar insectos en grupos dietarios, utilizando como base la revisión de su contenido estomacal (10). La información generada en este estudio llevado a cabo en la región, puede ser un referente más adecuado para la clasificación de entomofauna presente en otras quebradas andinas con condiciones similares.

La cuenca del río La Vieja presenta un sistema de pequeñas quebradas que drenan principalmente a través de sistemas ganaderos con diferentes grados de intervención. A pesar de que estas quebradas ya han sido objeto de algu- nos estudios sobre la composición de la fauna béntica y su interacción con el hábitat (e.g. 11-13), no se conocen investigaciones sobre las relaciones tróficas de estas comunidades acuáticas.

Esta investigación se realizó con el objetivo de determinar la estructura trófica de la entomofauna de ocho quebradas de la cuenca del río La Vieja, utilizando como base la clasificación en grupos dietarios de insectos de quebradas de la cuenca del río Otún (10).

\section{Materiales y métodos}

Se seleccionaron ocho quebradas en la cuenca del río La Vieja, ubicadas en los municipios de Alcalá, Ulloa y Cartago (Valle del Cauca), entre 4'38'29" y 441'46" Norte y 7543'00”' y 75'52'11.4" Oeste (Figura 1). Todas las quebradas son de primer orden y se localizan entre los 1115 y $1140 \mathrm{msnm}$, en microcuencas sin problemas de contaminación evidente, con cobertura de bosque secundario y cafetales. En la Tabla 1 se presenta un resumen de las características fisicoquímicas de las quebradas estudiadas.

El muestreo de macroinvertebrados se realizó con una red tipo "D" de $500 \mu \mathrm{m}$ de ojo de malla, mediante 20 arrastres en los hábitats más representativos en proporción a su ocurrencia. Los macroinvertebrados fueron conservados en etanol al $75 \%$ para su posterior identificación hasta el menor nivel taxonómico posible con ayuda de un estereoscopio y claves taxonómicas especializadas para los diferentes grupos (14). El trabajo taxonómico se llevó a cabo en el Laboratorio de Investigaciones Entomológicas de la Universidad del Valle y los ejemplares se encuentran depositados en el Museo de Entomología de esta institución (MUSENUV). 


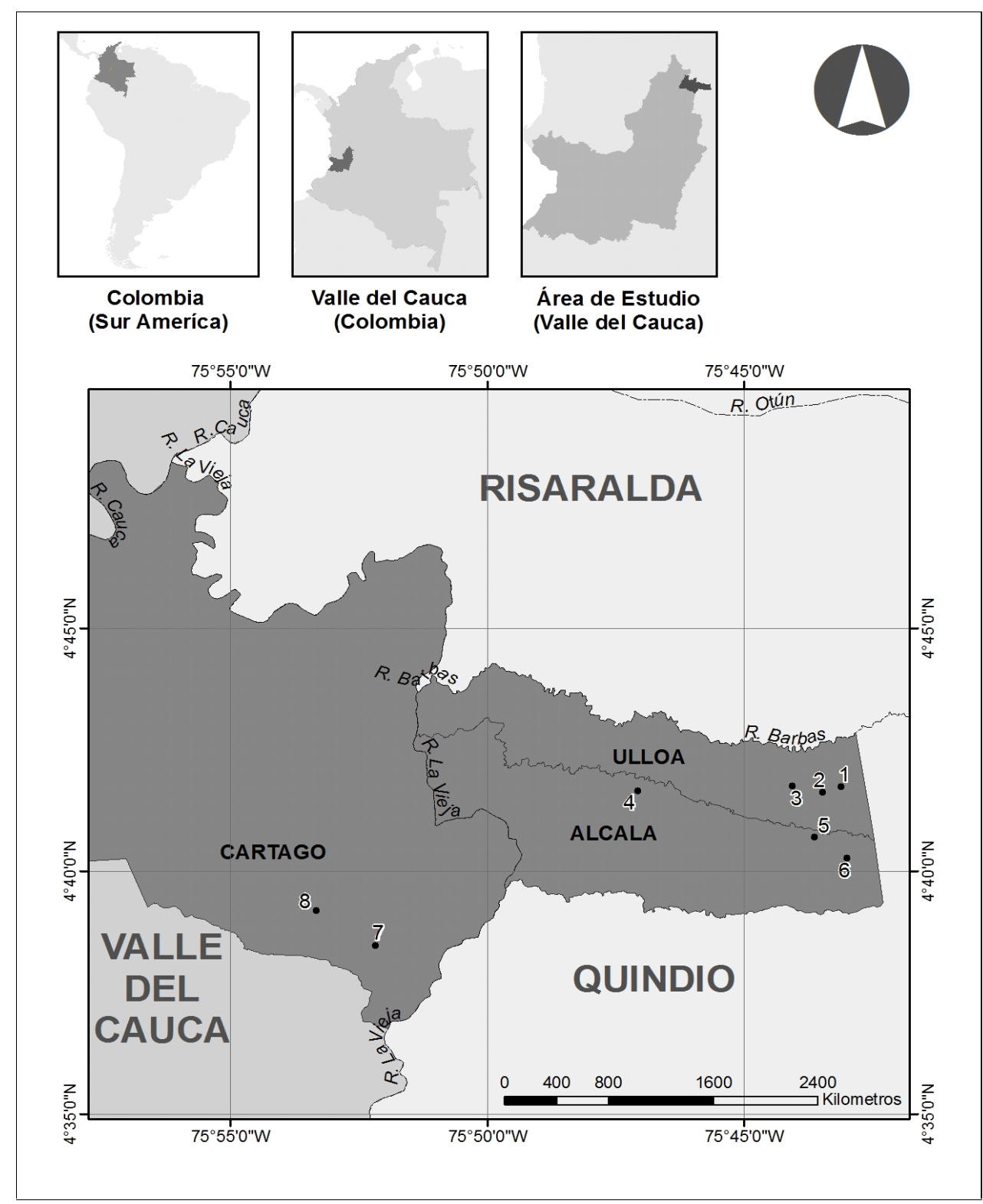

Figura 1. Área de estudio en la ecorregión cafetera. Ubicación de las quebradas protegidas muestreadas: 1) La Azulita, 2) El Bosque 3) El Recreo II, 4) La Frontera, 5) El Recreo I, 6) La Puerta de Alcalá, 7) El Guayabo, 8) NN.

Los insectos colectados en el muestreo fueron asignados a grupos tróficos con base en la clasificación propuesta por Chará-Serna et al. (10), que resulta de la revisión del contenido estomacal de insectos asociados a paquetes de hojarasca, en nueve quebradas de la cuenca del río Otún ubicadas entre los 900 y $2500 \mathrm{msnm}(10)$.

Estas corrientes se tomaron como referencia porque son de bajo orden, poseen corredores ribereños de bosque secundario, no son afectadas por problemas de contaminación directa y presentan una composición de insectos acuáticos similar a las quebradas que son objeto de estudio.
Se consideraron los siguientes grupos dietarios, a partir del porcentaje promedio de los tipos de alimento reportados en el contenido estomacal de cada taxón (10): 1) fragmentadores -contenido de materia orgánica particulada gruesa mayor o igual a $35 \%$; 2) colectores -contenido de materia orgánica particulada fina mayor o igual a $65 \%$; 3 ) depredadores -contenido de tejido animal mayor o igual a $35 \%$; 4) raspadores -contenido de algas mayor o igual a $35 \%$. Esta clasificación se complementó con literatura (2) para los taxa que no fueron registrados en el estudio mencionado. 
Tabla 1. Características físicas y de calidad de agua de ocho quebradas protegidas de la ecorregión cafetera

\begin{tabular}{lrrr}
\hline Parámetro & Promedio & Mínimo & Máximo \\
\hline Dimensiones del cauce & & & \\
Ancho banco (cm) & 119,6 & 0,0 & 272,0 \\
Ancho húmedo (cm) & 107,5 & 0,0 & 319,0 \\
Profundidad (cm) & 7,3 & 0,0 & 13,5 \\
Caudal (1/s) & 3,8 & 1,7 & 7,8 \\
Tipo de corriente & & & 20,0 \\
Turbulencias (\%) & 15,0 & 10,0 & 90,0 \\
Corriente rápida (\%) & 57,1 & 5,0 & 90,0 \\
Corriente lenta (\%) & 46,0 & 20,0 & 20,0 \\
Piscinas (\%) & 10,0 & 5,0 & 70,0 \\
Tipo de sustrato inorgánico & & & 20,0 \\
Piedras (mayor a 5 cm de diámetro) (\%) & 33,6 & 0,0 & 90,0 \\
Grava (0,2 cm 5 cm de diámetro) (\%) & 13,3 & 10,0 & 50,0 \\
Sustratos finos (menor a 0,2 cm de diámetro) (\%) & 55,0 & 20,0 & 40,0 \\
Tipo de sustrato orgánico & & & 137,0 \\
Hojarasca (\%) & 37,1 & 20,0 & 22,1 \\
Materia orgánica particulada gruesa (\%) & 22,0 & 10,0 & 8,4 \\
Índice de calidad de hábitat (ICH) & 125,1 & 102,0 & 11,4 \\
Calidad del agua & & & 1,0 \\
Temperatura del agua ( ${ }^{\circ}$ C) & 20,2 & 19,5 & 0,1 \\
pH & 6,9 & 5,9 & \\
Oxígeno disuelto (mg/l) & 5,7 & 1,9 & \\
DBO5 (mg/l) & 0,5 & 0,1 & 0,0 \\
Fosfatos (mg/l P-PO4) & 0,1 & 0,0 & \\
\hline
\end{tabular}

${ }^{1}$ Índice de Calidad de Agua de la Fundación Sanitaria Nacional de los Estados Unidos de Norteamérica (15).

\section{Resultados}

En total se colectaron 2019 insectos distribuidos en 73 taxa, de los cuales fue posible identificar nueve órdenes, 39 familias y 55 géneros. Los órdenes que presentaron mayor riqueza de géneros fueron: Trichoptera con 13, Odonata con 12, Coleoptera y Diptera con 11 y Ephemeroptera con 10 (Figura 2).

El taxón más numeroso fue Chironomidae con el 20,8\% de la abundancia relativa de la muestra, seguido por Smicridea con el $14,7 \%$ y Simulium con el $10,7 \%$ (Figura 3).

En la Tabla 2 se presenta la identificación taxonómica de los insectos colectados y su respectiva asignación a grupos dietarios; para los individuos que no fue posible identificar al nivel de género se reporta el grupo trófico predominante en la familia. 60 de los 73 taxa fueron clasi- ficados en grupos dietarios; 38 de acuerdo con la propuesta de Chará-Serna et al. (10) y 22 que no fueron registrados en ese estudio, se clasificaron según Merritt y Cummins (2). Los colectores fueron el grupo dietario más importante, representando el 55\% de la muestra. En segundo lugar se ubicaron los fragmentadores con el $31 \%$. Los depredadores agruparon el $10 \%$ de los insectos colectados y Psephenus fue el único género asignado a la categoría de raspadores $(0,05 \%)$.

Los colectores, el grupo dietario más abundante de la comunidad estuvo conformado por 23 taxa (5 órdenes, 15 familias, 17 géneros), entre los que Chironomidae, Simulium y Heterelmis fueron los más importantes, constituyendo el $41,5 \%$ de la muestra total. Esta categoría también incluyó otros géneros relevantes en términos de abundancia tales como Leptohyphes, Farrodes y Macrelmis. 


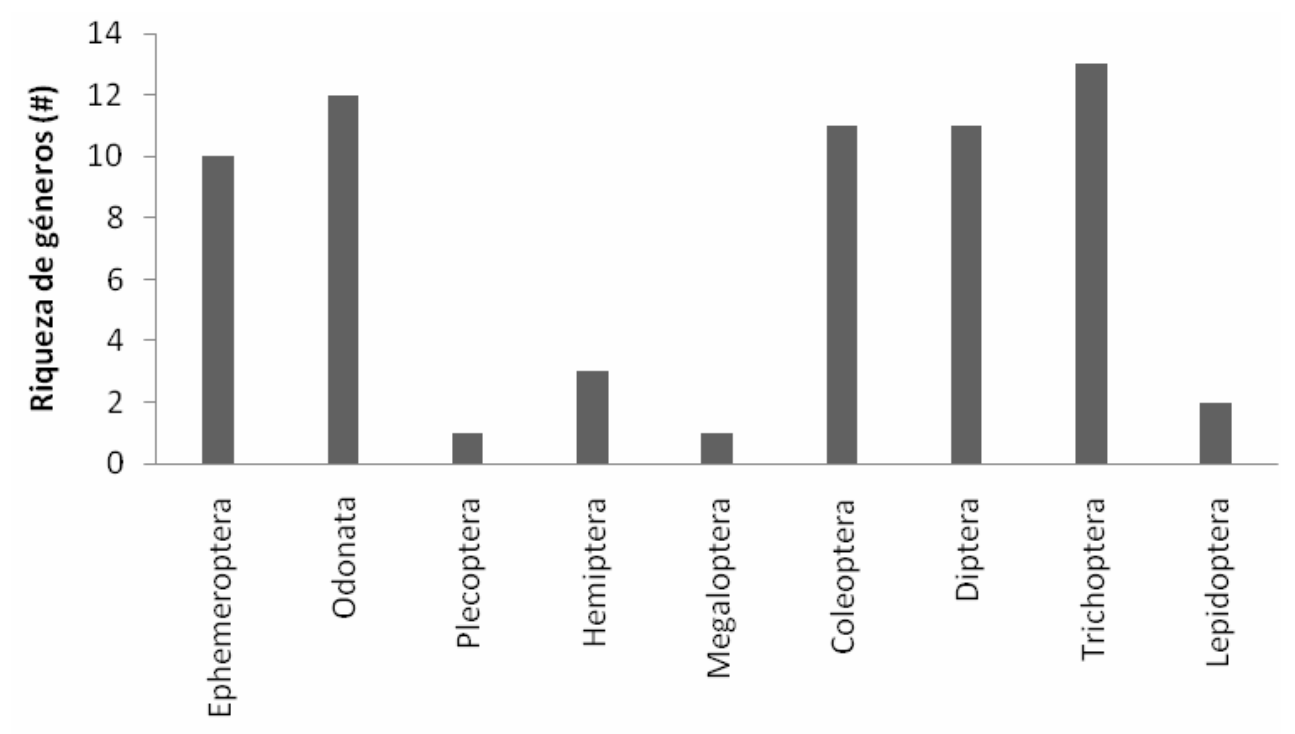

Órdenes de Insecta

Figura 2. Riqueza de géneros por cada orden de insectos presente en las quebradas protegidas de la ecorregión cafetera muestreadas.

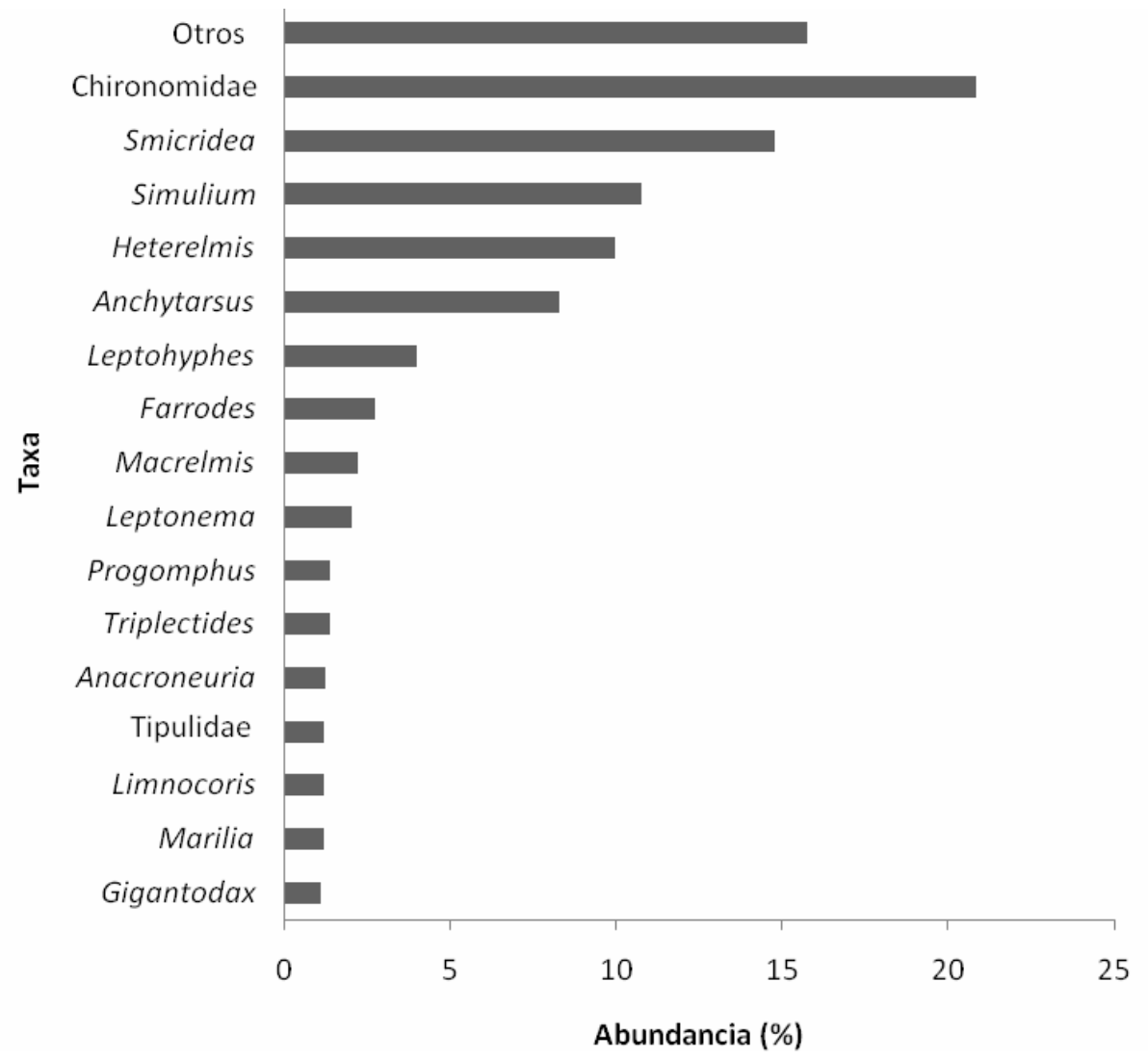

Figura 3. Abundancia relativa de los grupos taxonómicos más importantes encontrados en quebradas protegidas de la ecorregión cafetera. 
Tabla 2. Clasificación en grupos dietarios de los insectos colectados en las quebradas protegidas muestreadas de la ecorregión cafetera.

\begin{tabular}{|c|c|c|c|}
\hline Orden & Familia & Género & Grupo dietario \\
\hline \multirow[t]{10}{*}{ Ephemeroptera } & Baetidae & & Colector $^{2}$ \\
\hline & & Americabaetis & Colector $^{1}$ \\
\hline & & Baetodes & Colector $^{1}$ \\
\hline & & Camelobaetidius & N.I. \\
\hline & & Zelusia & N.I. \\
\hline & Leptohyphidae & Haplohyhes & Colector $^{1}$ \\
\hline & & Leptohyphes & Colector $^{1}$ \\
\hline & & Tricorythodes & Fragmentador ${ }^{1}$ \\
\hline & Leptophlebiidae & Farrodes & Colector $^{1}$ \\
\hline & & Thraulodes & Colector $^{1}$ \\
\hline Plecoptera & Perlidae & Anacroneuria & Depredador $^{1}$ \\
\hline Megaloptera & Corydalidae & Corydalus & Depredador ${ }^{2}$ \\
\hline \multirow[t]{12}{*}{ Trichoptera } & Calamoceratidae & Phylloicus & Fragmentador ${ }^{1}$ \\
\hline & Helicopsychidae & Helicopsyche & Colector $^{1}$ \\
\hline & Hydrobiosidae & Atopsyche & Depredador $^{1}$ \\
\hline & Hydropsychidae & Leptonema & Fragmentador ${ }^{1}$ \\
\hline & & Smicridea & Fragmentador $^{1}$ \\
\hline & Leptoceridae & Atanatolica & Colector $^{1}$ \\
\hline & & Nectopsyche & Fragmentador ${ }^{1}$ \\
\hline & & Oecetis & Depredador $^{1}$ \\
\hline & & Triplectides & Fragmentador $^{1}$ \\
\hline & Odontoceridae & Marilia & Fragmentador $^{1}$ \\
\hline & Philopotamidae & Chimarra & N.I. \\
\hline & Polycentropodidae & Polycentropus & Depredador $^{1}$ \\
\hline \multirow{12}{*}{ Odonata } & Gomphidae & & Depredador $^{2}$ \\
\hline & & Progomphus & Depredador ${ }^{1}$ \\
\hline & Libellulidae & & Depredador ${ }^{1}$ \\
\hline & & Brechmorhoga & Depredador $^{2}$ \\
\hline & & Cannaphila & Depredador $^{2}$ \\
\hline & & Dythemis & Depredador ${ }^{2}$ \\
\hline & Calopterygidae & & Depredador ${ }^{1}$ \\
\hline & & Hetaerina & Depredador $^{1}$ \\
\hline & Coenagrionidae & & Depredador $^{2}$ \\
\hline & & Argia & Depredador $^{2}$ \\
\hline & Megapodagrionidae & & Depredador ${ }^{1}$ \\
\hline & Polythoridae & & Depredador ${ }^{1}$ \\
\hline \multirow[t]{3}{*}{ Hemiptera } & Gerridae & Limnogonus & Depredador $^{2}$ \\
\hline & Naucoridae & Limnocoris & Depredador ${ }^{2}$ \\
\hline & Veliidae & Rhagovelia & Colector $^{1}$ \\
\hline \multirow[t]{2}{*}{ Lepidoptera } & Pyralidae & & Fragmentador $^{2}$ \\
\hline & & Crambus & N.I. \\
\hline \multirow[t]{5}{*}{ Coleoptera } & Dytiscidae & & Depredador $^{2}$ \\
\hline & Elmidae & Heterelmis (adulto) & Colector $^{1}$ \\
\hline & & Hexacylloepus & N.I. \\
\hline & & Macrelmis (adulto) & Colector $^{1}$ \\
\hline & & Microcylloepus & N.I. \\
\hline
\end{tabular}


Continuación Tabla 2

\begin{tabular}{|c|c|c|c|}
\hline Orden & Familia & Género & Grupo dietario \\
\hline \multirow{28}{*}{ Diptera } & Lampyridae & & N.I. \\
\hline & Psephenidae & Psephenus & Raspador $^{2}$ \\
\hline & Ptilodactylidae & Anchytarsus & Fragmentador ${ }^{1}$ \\
\hline & Scirtidae & Elodes & N.I. \\
\hline & Staphylinidae & Eleusis & N.I. \\
\hline & & Lissohypus & N.I. \\
\hline & Ceratopogonidae & & Depredador $^{2}$ \\
\hline & & Atrichopogon & Colector $^{1}$ \\
\hline & & Culicoides & Depredador $^{2}$ \\
\hline & & Ceratopogon & N.I. \\
\hline & Chironomidae & & Colector $^{2}$ \\
\hline & Dixidae & & Colector $^{1}$ \\
\hline & & Dixella & Colector $^{1}$ \\
\hline & Empididae & & Fragmentador ${ }^{1}$ \\
\hline & Sciomyzidae & & Depredador $^{2}$ \\
\hline & Simuliidae & Gigantodax & N.I. \\
\hline & & Simulium & Colector $^{1}$ \\
\hline & Stratiomyidae & & Colector $^{1}$ \\
\hline & & Allognosta & N.I. \\
\hline & & Odontomyia & Colector $^{2}$ \\
\hline & & Stratiomys & Colector $^{2}$ \\
\hline & Tipulidae & & Fragmentador $^{2}$ \\
\hline & & Gonomyia & N.I. \\
\hline & & Hexatoma & Depredador $^{1}$ \\
\hline & & Limonia & Fragmentador $^{2}$ \\
\hline & & Tipula & Fragmentador ${ }^{1}$ \\
\hline & Psychodidae & & Colector $^{2}$ \\
\hline & & Pericoma & Colector $^{2}$ \\
\hline
\end{tabular}

${ }^{1}$ Clasificados según Chará-Serna et al. (10), ${ }^{2}$ Clasificados según Merritt y Cummins (2). N.I.: Sin información.

Los fragmentadores agruparon 13 taxa (4 órdenes, 9 familias, 17 géneros). Los más abundantes fueron Smicridea y Anchytarsus con el 23\% de la muestra total. Otros taxa significativos en este grupo dietario fueron Leptonema, Marilia, Triplectides y Tipulidae.

El grupo dietario depredadores presentó 24 taxa distribuidos en siete órdenes, 19 familias y 16 géneros. No obstante los grupos taxonómicos de esta categoría presentaron abundancias inferiores a 30 individuos. Los taxa más importantes en este grupo fueron Progomphus, Anacroneuria y Limnocoris.

\section{Discusión}

La composición trófica de las comunidades de insectos examinadas en el presente estudio presentó cierto grado de similitud con la reportada por Chará et al. (10) en quebradas de la cuenca del río Otún. En ambos casos el grupo dietario más abundante fue el de los colectores, seguido por los fragmentadores y en tercer lugar los depredadores. Resultados afines fueron reportados en una quebrada altoandina ecuatoriana con cobertura boscosa, en la que los colectores fueron dominantes y había un porcentaje importante de fragmentadores (16).

Estas similitudes en la estructura trófica sugieren un patrón de funcionamiento de las comunidades de insectos presentes en quebradas andinas con bosques ribereños, que coincide con el concepto de río continuo (17). Según éste la codominancia de fragmentadores y colectores (que se alimentan de hojarasca y materia orgánica particulada fina derivada de la misma) refleja la importancia de este recurso, en quebradas de cabecera con cobertura boscosa, donde la fotosíntesis es limitada 
por la sombra $(17,18)$. Esta limitación define a estos ecosistemas como heterotróficos y explicaría la escasa presencia de taxa raspadores, cuya principal fuente de alimento son las algas.

La estructura trófica de estas quebradas andinas, donde la abundancia de fragmentadores implica que estos insectos tienen un papel importante en la descomposición de hojarasca, concuerda con la registrada en varias investigaciones llevadas a cabo en quebradas tropicales $(8,19,20,21)$. Sin embargo, estos resultados contrastan con otros estudios que reportan una marcada escasez de fragmentadores, argumentando que la descomposición de la hojarasca por microorganismos en estas latitudes es acelerada debido a las altas temperaturas y remplaza a la causada por invertebrados (e.g. 22, 23, 24). Estas diferencias pueden explicarse por la gran heterogeneidad que presentan los ecosistemas tropicales y por el uso de clasificaciones generadas en zonas templadas, que inducen errores en la asignación de taxa a gremios tróficos $(8,16)$.

Otra tendencia interesante registrada en estudios de quebradas protegidas de los Andes colombianos (10, 25), es que a pesar de la dominancia numérica de los colectores, los fragmentadores son más importantes en términos de biomasa por tratarse de individuos de mayor tamaño. Por esta razón sería recomendable evaluar la biomasa relativa de los grupos dietarios de la cuenca del río La Vieja, con el fin de establecer si se mantiene esta condición, que confirma la relevancia ecológica de los fragmentadores. Esto es probable porque los colectores fueron conformados principalmente por taxa de tamaños reducidos como Chironomidae, Simulium y Heterelmis; mientras los fragmentadores como Smicridea, Anchytarsus, Leptonema y Tipulidae, por lo general presentaron tallas relativamente mayores.

Es necesario tener en cuenta que la clasificación en grupos dietarios basada en resultados obtenidos en quebradas andinas de condiciones similares (riberas boscosas, ausencia de signos evidentes de contaminación), sólo constituye un acercamiento a la ecología de estos insectos tan poco estudiados en el país. Se recomienda analizar el contenido estomacal de algunos representantes de cada taxón colectado para confirmar la asignación a grupos dietarios propuesta en este estudio, ya que no se descarta la posibilidad de que el hábito alimenticio de los taxa varíe entre las dos cuencas. Resultados discrepantes en la asignación a grupos funcionales de insectos han sido reportados en quebradas andinas ecuatorianas (16) y bolivianas (26), aún cuando en ambos casos se utilizó la revisión de contenido estomacal como método de clasificación (16). Estas variaciones son un ejemplo de la alta plasticidad que exhiben los taxa neotropicales en sus hábitos alimenticios, característica que les facilita adaptarse a las condiciones fluctuantes del trópico (26).

El presente trabajo evidencia el desconocimiento que existe sobre la ecología de los organismos que habitan las quebradas tropicales: el 50\% de los grupos taxonómicos reportados no presentaron información de hábitos dietarios en la zona tropical, cifra que incluye a un $20 \%$ de taxa para los que no existe información ni en el trópico ni en la zona templada. Este hecho destaca la necesidad de continuar profundizando en los aspectos ecológicos de las quebradas andinas, que a pesar del impacto que han sufrido por las actividades humanas, siguen constituyendo un refugio significativo de diversidad, como lo indica la cantidad de géneros de insectos acuáticos identificados en ellas.

\section{Conclusiones}

La comunidad de insectos acuáticos de las quebradas estudiadas en la cuenca del río La Vieja está compuesta principalmente por colectores, fragmentadores y depredadores. Los grupos con mayor cantidad de individuos fueron los dos primeros, que sumaron el $86 \%$ de la abundancia.

Este hecho confirma la importancia de la materia orgánica particulada gruesa (hojarasca) como recurso alimenticio para la comunidad de insectos estudiada y, por ende, como fuente de energía en estos ambientes acuáticos.

La similitud entre la estructura trófica de las comunidades estudiadas respecto a otras reportadas para la zona andina, sugieren un patrón de funcionamiento ecológico de las quebradas andinas con bosques ribereños.

Se recomienda la evaluación de la biomasa relativa de los grupos dietarios, para determinar si los fragmentadores son dominantes, tal como lo sugieren las tallas características de los taxa que conformaron este grupo dietario en el presente estudio.

Existe un gran desconocimiento sobre el funcionamiento de las quebradas tropicales, razón por la cual es fundamental la realización de más estudios de tipo ecológico, que permitan una mejor comprensión de los procesos que ocurren en estos ecosistemas y la influencia que las actividades humanas ejercen sobre éstos. 


\section{Agradecimientos}

Los autores agradecen el apoyo brindado por el Proyecto "Enfoques silvopastoriles integrados para el manejo de ecosistemas" financiado por el GEF-Banco Mundial y FAOLEAD, al Laboratorio de Investigaciones Entomológicas de la Universidad del Valle por el apoyo logístico en el trabajo de identificación taxonómica, a Santiago Santacruz y Lucimar Dias por sus valiosos aportes al manuscrito, y a Carlos Ríos de la Fundación Wildlife Conservation Society (WCS) Programa Colombia por la elaboración del mapa.

\section{Financiación}

Esta investigación fue financiada parcialmente por el Centro de Investigaciones y Estudios en Biodiversidad y Recursos Genéticos, CIEBREG. El trabajo hace parte del proyecto "Desarrollo de servicios ambientales en paisajes ganaderos de la cuenca del río La Vieja", ejecutado por la Fundación Centro para la Investigación en Sistemas Sostenibles de Producción Agropecuaria-CIPAV, en convenio con la Corporación Autónoma Regional del Valle del Cauca-CVC. Además contó con aportes del Convenio COLCIENCIAS-SENA a CIPAV (mediante contrato 4802008), del programa “Jóvenes Investigadores e Innovadores año 2008" de COLCIENCIAS y de la Fundación Internacional para la Ciencia (IFS).

\section{Conflictos de intereses}

Los autores afirman no tener conflictos de intereses.

\section{Referencias}

1. Yule CM. Trophic relationships and food webs of the benthic invertebrate fauna of two aseasonal tropical streams in Bougainville Island, Papua New Guinea. Journal of Tropical Ecology. 1996; 12: 517-534.

2. Merrit $\mathrm{RW}$, Cummins $\mathrm{KW}$. An Introduction to the Aquatic Insects of North America. Third edition. Kendall-Hunt Publishing Company. Iowa, USA. 1996; 862 p.

3. Sabater S, Elosegi A, Acuña V, Basaguren A, Muñoz I, Pozo J. Effect of climate on the trophic structure of temperate forested streams. A comparison of Mediterranean and Atlantic streams. Science of the Total Environment. 2008; 390: 475-484.
4. Zilli FL, Montalto L, Marchese MR. Benthic invertebrate assemblages and functional feeding groups in the Paraná River floodplain (Argentina). Limnologica. 2008; 38: 159-171.

5. Mathuriau C, Chauvet E. Breakdown of leaf litter in a neotropical stream. Journal of the North American Benthological Society. 2002; 21 (3): 384-396.

6. Rueda-Delgado GK, Wantzen M, Tolosa MB. LeafLitter decomposition in an Amazonian floodplain stream: effects of seasonal hydrological changes. Journal of the North American Benthological Society. 2006; 25: 233-249.

7. Gonçalves JF, Graça MAS, Callisto M. Leaf-litter breakdown in 3 streams in temperate, Mediterranean, and tropical Cerrado climates. Journal of the North American Benthological Society. 2006; 25 (2): 344355.

8. Cheshire L, Boyero L, Pearson RG. Food webs in tropical Australian streams: shredders are not scarce. Freshwater Biology. 2005; 50: 748-769.

9. Dobson M, Magana A, Mathooko JM, Ndegwa FK. Detritivores in Kenyan highland streams: more evidence for the paucity of shredders in the tropics? Freshwater Biology. 2002; 47: 909-919.

10. Chará-Serna AM, Chará J, Zúñiga MC, Pearson RG, Boyero L. Diets of leaf-litter-associated insects in three Colombian streams (en prensa).

11. Chará J, Zúñiga MC, Giraldo LP, Pedraza G, Astudillo M, Ramírez L, Posso CE. Diversidad y abundancia de macroinvertebrados acuáticos en quebradas de la cuenca del río La Vieja, Colombia. En: Rodríguez JM, Camargo JC, Niño J, Pineda AM, Arias LM, Echeverry MA, Miranda CL. (eds.). Ciebreg, Valoración de la biodiversidad en la ecorregión del eje cafetero. Ciebreg. Pereira, Colombia. 2009; 127-142.

12. Pedraza GX, Giraldo LP, Chará J. Efecto de la restauración de corredores ribereños sobre características bióticas y abióticas de quebradas en zonas ganaderas de la cuenca del río La Vieja, Colombia. Zootecnia Tropical. 2008; 26 (3): 1-4.

13. Gutiérrez-Chacón C, Zúñiga MC, Van Bodegom PM, Chará J, Giraldo L. Rove beetles (Coleoptera: Staphylinidae) in neotropical riverine landscapes: characterising their distribution. Insect conservation and diversity. 2009; 2: 106-115.

14. Fernández HR, Domínguez E. Guía para la determinación de los artrópodos bentónicos sudamericanos. Primera edición. Universidad Nacional de Tucumán. Tucumán, Argentina. 2001, 282 p. 
15. Dinius SH. Design of a water quality index. Water research bulletin. 1987; 23: 883-843.

16. Rios B, Encalada A, Prat N. Leaf litter dynamics and its use by invertebrates in a high-altitud tropical Andean stream. International Review of Hydrobiology. 2009; 94 (4): 357-371.

17. Vannote RL, Minshall GW, Cummins KW, Sedell JR, Cushing CE. The river continuum concept. Canadian Journal of Fisheries and Aquatic Science. 1980; 37: 130-137.

18. Allan JD. Stream Ecology: Structure and Function of Running waters. First edition. Kluwer Academic Publishers. Dordrecht, Holanda. 1995; 388 p.

19. Wright MS, Covich AP. The effect of macroinvertebrate exclusion on leaf breakdown rates in a tropical headwater stream. Biotropica. 2005; 37: 403-408.

20. Yule CM, Leong MY, Liew KC, Ratnarajah L, Schmidt K, Wong HM, Pearson RG, Boyero L. Shredders in Malaysia: Abundance and species richness are higher in highland, temperate-like, tropical streams. Journal of the North American Bentholgical Society. 2009; 28: 404-415.

21. Camacho R, Boyero L, Cornejo A, Ibáñez A, Pearson RG. Local variation in shredder numbers can explain their oversight in tropical streams. Biotropica. 2009; 41 (5): 625-632.

22. Dobson M, Magana A, Mathooko JM, Ndegwa FK. Detritivores in Kenyan highland streams: more evidence for the paucity of shredders in the tropics? Freshwater Biology. 2002; 47: 909-919.

23. Rueda-Delgado GK, Wantzen M, Tolosa MB. LeafLitter decomposition in an Amazonian floodplain stream: effects of seasonal hydrological changes. Journal of the North American Benthological Society. 2006; 25: 233-249.

24. Wantzen KM, Wagner R. Detritus processing by invertebrate shredders: a neotropical-temperate comparison. Journal of the North American Benthological Society. 2006; 25: 216-232.

25. Chará J, Baird D, Telfer T, Giraldo L. Litter degradation of three native leaf species in a slow-flow headwater stream in Colombia. International Review of Hydrobiology. 2007; 92: 183-198.

26. Tomanova S, Goitia E, Helešic J. Trophic levels and functional feeding groups of macroinvertebrates in neotropical streams. Hydrobiology. 2006; 556: 251264. 